\title{
Microchimica Acta topical collection IX NyNA 2019
}

\author{
Juan R. Castillo ${ }^{1}$ - Alberto Escarpa ${ }^{2,3}$
}

Published online: 2 May 2020

(C) Springer-Verlag GmbH Austria, part of Springer Nature 2020

In 2006, Spanish researchers in analytical chemistry launched a pioneering initiative: the first workshop on Analytical Nanoscience and Nanotechnology. Initially at the national level, the objective of the workshop was to bring together Spanish scientists interested in analytical nanoscience and nanotechnology, enabling them to know who was who and to establish collaborations and links in a research topic which at that time was considered a rising star in the field.

From the beginning, the main goal of this conference was to promote the development of novel approaches at the interface between analytical chemistry and nanoscience and nanotechnology within the framework of nanomaterials as analytes in single and complex matrices, mainly as tools for developing analytical detection principles and methods. Since then, nine events took place.

We are delighted to introduce the first Microchimica Acta Topical Collection with selected papers from the latest proceedings of this International Congress on Analytical Nanoscience and Nanotechnology (IX NyNA 2019, Zaragoza, Spain). The selected works demonstrate how nano- and micro-materials offer unique opportunities to overcome analytical challenges, from the analysis of contaminants

This editorial corresponds to the IX NyNA 2019, International Congress on Analytical Nanoscience and Nanotechnology at Zaragoza (Spain) on 2-4 July 2019

Juan R. Castillo

jcastilo@unizar.es

Alberto Escarpa

alberto.escarpa@uah.es

1 Environmental Sciences Institute, Department of Analytical Chemistry, Faculty of Sciences, University of Zaragoza, Zaragoza, Spain

2 Department of Analytical Chemistry, Physical Chemistry and Chemical Engineering, Faculty of Sciences Universidad de Alcalá, Ctra.Madrid-Barcelona, Km. 33.600, 28871 Alcalá de Henares, Madrid, Spain

3 Chemical Research Institute "Andres M. Del Rio", Universidad de Alcalá, Madrid, Spain to monitoring of biomarkers, to cell studies. In this regard, noble metals, colloidal nanoparticles, and nanostructures and nanocomposite materials once again demonstrate their feasibility as unique tools for the scientific community, ready to use in solving complex challenges.

Silver has been employed under different dimensions and conformations, from colloidal dispersions to microstructures, confirming a renaissance of the 'silver age'. Threedimensional silver mirror-like micro-pyramids are employed as an active substrate for surface-enhanced Raman spectroscopy (SERS), enabling the ultrasensitive determination of organophosphorus pesticides [1]. Heterocyclic aromatic amines are determined in food samples using a screen-printed carbon nanofiber electrode modified with silver nanoparticles (AgNPs) embedded in the Nafion matrix. The proposed sensor coupled with liquid-liquid microextraction assisted by ionic liquid was applied for the determination of 2-amino-3,8dimethylimidazo[4,5-f]quinoxaline [2]. A sensor based on Lcysteine oligomers electropolymerized on a glassy carbon electrode is proposed for the rapid quantification of AgNPs. Ligands with amino, sulfur and carboxy functional groups are used to retain AgNPs, followed by stripping voltammetry [3]. A method for quantifying water-stabilized silver sulfide quantum dots further illustrates the suitability of stripping voltammetry for the determination of such tiny nanotools. Escherichia coli determination, where silver sulphide quantum dots are used as tags, is employed as a proof of concept to demonstrate the outstanding applications of the method [4].

Gold nanoparticles (AuNP) still remain unique tools, having earned a place of honor in the ecosystem for the use of nanomaterials for analytical purposes. Our article selection illustrates the unprecedented capability of AuNPs as replacement for $\mathrm{O}_{2}$ in the oxidase enzymatic reaction. This strategy is employed to determine tyramine in cheese samples using tyramine oxidase and exploiting the localized surface plasmon resonance (LSPR) of the formed AuNPs as analytical signals [5]. Taking further advantage of the AuNPs LSPR, a colorimetric assay is proposed for microRNA146a determination. AuNPs functionalized with selected RNA probes aggregate in the presence of miR146a, resulting in a color/plasmonic shift 
used as analytical signal, with excellent performance and applicability for the analysis of raw cow milk samples [6].

The topical collection also covers novel developments in not so widely used nanomaterials. For example, $\mathrm{ZnO}$ nanowires are employed for the determination of lactate and cholesterol through the detection of hydrogen peroxide generated during the enzymatic reactions (using lactate oxidase and cholesterol oxidase), taking advantage of the electrostatic interactions between the enzymes and the $\mathrm{ZnO}$ nanowires. By exploiting the ability of hydrogen peroxide to quench the fluorescence of the $\mathrm{ZnO}$ nanowires, lactate and cholesterol are determined [7].

Exploiting magnetic beads, and using a screen-printed carbon electrode, an immunosensor is proposed for the analysis of HER2-ECD, a biomarker in breast cancer and breast cancer cells in human serum. The affinity process is detected using electroactive labels composed of core/shell streptavidin-modified CdSe@ZnS quantum dots. $\mathrm{Cd}^{2+}$ ions released from the quantum dots are determined by differential pulse anodic stripping voltammetry. The immunoassay was successfully applied to breast cancer cell lines SK-BR-3 (a HER2-positive cell line), MDAMB-231 (a HER2-negative cell line), and MCF-7 (a cell line with low HER2 expression) [8]. A method for the electrochemical detection of methylisothiazolinone is also proposed, taking advantage of membranes [polystyrene sulfonate or poly(diallyldimethylammonium)] incorporating AuNPs [9].

In sum, the selected manuscripts reflect the 'horizontal use' of nanomaterials, involving different analytical fields and exciting applications.

This topical collection demonstrates how the IX NyNA 2019 conference has again proved to be a fruitful occasion of high-level scientific debate and vibrant activities of Spanish analytical nanoscience.

We are looking forward for the next edition that will take place in 2021 in Spain.

We invite our entire readership to participate in this congress and to send their best works in the field of analytical sciences based on micro- and nanomaterials to the journal.

We hope our readership enjoys this collection and finds it useful.

Juan R. Castillo,

Guest Editor

Alberto Escarpa,

Editor in Chief, Microchimica Acta

\section{Compliance with ethical standards}

Conflict of interest The authors declare that they have no competing interests.

\section{References}

1. Lafuente M, Berenschot E, Tiggelaar R, Mallada R, Tas N, Pina MP (2020) Attomolar SERS detection of organophosphorous pesticides using silver mirror like micro-pyramids as active substrate. Microchim Acta 187:247. https://doi.org/10.1007/s00604-0204216-9

2. Montes C, Contento AM, Villaseñor MJ, Ríos Á (2020) A screenprinted electrode modified with silver nanoparticles and carbon nanofibers in a nafion matrix for ionic liquid-based dispersive liquid-liquid microextraction and voltammetric assay of heterocyclic amine 8-MeIQx in food. Microchim Acta 187:190. https://doi.org/ 10.1007/s00604-020-4138-6

3. Vidal JC, Torrero D, Menés S, Fuente A, Castillo JR (2020) Voltammetric sensing of silver nanoparticles on electrodes modified with selective ligands by using covalent and electropolymerization procedures. Discrimination between silver(I) and metallic silver. Microchim Acta 187:183. https://doi.org/10.1007/s00604-0204139-5

4. Amor-Gutiérrez O, Iglesias-Mayor A, Llano-Suárez P, CostaFernández JM, Soldado A, Podadera A, Parra F, Costa-García A, Escosura-Muñiz A (2020) Electrochemical quantification of $\mathrm{Ag}_{2} \mathrm{~S}$ quantum dots: evaluation of different surface coating ligands for bacteria determination. Microchim Acta 187:169. https://doi.org/ 10.1007/s00604-020-4140-z

5. Navarro J, de Marcos S, Galbán J (2020) Colorimetric-enzymatic determination of tyramine by generation of gold nanoparticles. Microchim Acta 187:174. https://doi.org/10.1007/s00604-0204141-y

6. Sánchez-Visedo A, Gallego B, Royo LJ, Soldado A, Valledor M, Ferrero FJ, Campo JC, Costa-Fernández JC, Fernández-Argüelles MT (2020) Visual detection of microRNA146a by using RNAfunctionalized gold nanoparticles. Microchim Acta 187:192. https://doi.org/10.1007/s00604-020-4148-4

7. Briones M, Busó-Rogero C, Catalán-Gómez S, García-Mendiola T, Pariente F, Redondo-Cubero A, Lorenzo ME (2020) ZnO nanowirebased fluorometric enzymatic assays for lactate and cholesterol. Microchim Acta 187:180. https://doi.org/10.1007/s00604-0204137-7

8. Freitas M, Nouws HPA, Keating E, Cruz Fernandes V, DelerueMatos C (2020) Immunomagnetic bead-based bioassay for the voltammetric analysis of the breast cancer biomarker HER2-ECD and tumour cells using quantum dots as detection labels. Microchim Acta 187:184. https://doi.org/10.1007/s00604-0204156-4

9. Abad-Gil L, Gismera MJ, Sevilla MT, Procopio JR (2020) Methylisothiazolinone response on disposable electrochemical platforms modified with carbon, nickel or gold-based nanomaterials. Microchim Acta 187:199. https://doi.org/10.1007/s00604-0204157-3

Publisher's note Springer Nature remains neutral with regard to jurisdictional claims in published maps and institutional affiliations. 